\title{
Audiometric outcomes of topical IGF1 treatment for sudden deafness refractory to systemic steroids.
}

\section{AUTHOR(S):}

Nakagawa, Takayuki; Ogino-Nishimura, Eriko;

Hiraumi, Harukazu; Sakamoto, Tastunori;

Yamamoto, Norio; Ito, Juichi

\section{CITATION:}

Nakagawa, Takayuki ...[et al]. Audiometric outcomes of topical IGF1 treatment for sudden deafness refractory to systemic steroids.. Otology \& neurotology 2012, 33(6): 941-946

ISSUE DATE:

2012-08

URL:

http://hdl.handle.net/2433/178678

\section{RIGHT:}

(C) 2012 Otology \& Neurotology, Inc.; This is not the published version.

Please cite only the published version.; この論文は出版社版でありませ ん。引用の際には出版社版をご確認ご利用ください。 


\section{Audiometric Outcomes of Topical IGF1 Treatment for Sudden Deafness \\ Refractory to Systemic Steroids}

Takayuki Nakagawa, MD, PhD; Eriko Ogino-Nishimura, MD; Harukazu Hiraumi, MD,

$\mathrm{PhD}$; Tastunori Sakamoto, MD, PhD; Norio Yamamoto, MD, PhD; Juichi Ito, MD, PhD

Department of Otolaryngology, Head and Neck Surgery, Graduate School of Medicine, Kyoto University, Kyoto, Japan

Running head: Topical IGF1 therapy for sudden deafness

Corresponding author: Takayuki Nakagawa

Department of Otolaryngology, Head and Neck Surgery,

Graduate School of Medicine, Kyoto University,

Kawaharacho 54, Shogoin, Sakyo-ku, 606-8507 Kyoto, Japan

Tel: +81-75-751-3346 Fax: +81-75-751-7225

E-mail: tnakagawa@ent.kuhp.kyoto-u.ac.jp

Conflicts of Interest and Source of Funding: This study was supported by grants for Research on Sensory and Communicative Disorders from the Japanese Ministry of Health, Labour and Welfare. The authors declare no conflicts of interest. 


\begin{abstract}
Objective: To examine the effects of topically applied recombinant human insulin-like growth factor 1 (IGF1) via gelatin hydrogels on alterations in pure-tone audiometry (PTA) in patients with sudden sensorineural hearing loss (SSHL) refractory to systemic steroids.
\end{abstract}

Study Design: Retrospective chart review. Setting: A single University Hospital.

Patients: Twenty-five patients with SSHL refractory to systemic steroids who received topical IGF1 treatment.

Intervention: Single topical application of IGF1 to the round window niche using gelatin hydrogels.

Main Outcome Measures: The primary outcome was alterations in PTA thresholds at frequencies of $0.25,0.5,1,2$ and $4 \mathrm{kHz}$ following topical IGF1 application. Secondary outcomes included differences in final improvements in PTA thresholds among frequencies tested and cumulative numbers of patients showing $10-$ or $20-\mathrm{dB}$ recovery in PTA during the observation period.

Results: Topical IGF1 application via gelatin hydrogels significantly altered PTA thresholds at each frequency tested and at the average frequency. The numbers of patients with a 10-dB recovery in PTA increased until four weeks after treatment and then stabilized, while those patients showing 20-dB recovery gradually increased during the observation period.

Conclusions: Topical IGF1 application via gelatin hydrogels contributes to the recovery of PTA levels in patients with SSHL refractory to systemic steroids. Major recovery of 
PTA levels occurs within four weeks after treatment. 


\section{INTRODUCTION}

Sudden sensorineural hearing loss (SSHL) is the most common cause of acute sensorineural hearing loss and is one of the few types that can be cured, with spontaneous recovery occurring in $30-60 \%$ of cases $(1,2)$. Most recoveries occur within the first two weeks of onset, and, after this time, it can be difficult to detect further improvement in hearing. The most common therapy for SSHL is the systemic application of glucocorticoids $(3,4)$, but about $20 \%$ of patients do not respond to this treatment (5). In addition, no therapy has been demonstrated to be effective according to the evidence criteria (6).

Various experimental studies have been performed to identify alternative therapeutics for acute sensorineural hearing loss including SSHL, and, of several potential candidates, insulin-like growth factor 1 (IGF1) was shown to be crucial for the development and maintenance of the cochlea (7); its clinical use has also been permitted, so it is a reasonable treatment for us to focus on. IGF1 delivery to the cochlear fluid at appropriate concentrations for sufficient durations has proved difficult, however, as most drugs, particularly growth factors, require sustained delivery for their appropriate actions. Topical application can increase drug concentrations in the cochlear fluid, but simple tympanic injection does not achieve sustained delivery (8). To overcome this, we have utilized gelatin hydrogels for the sustained release of proteins or peptides in topical cochlear treatment $(9,10)$.

Based on our pre-clinical studies (11-13), we performed a prospective clinical trial to test the safety and efficacy of topical IGF1 application via gelatin hydrogels for SSHL refractory to systemic steroids (14). The main outcome measures of this clinical 
trial were the proportions of patients showing hearing improvement, as determined by hearing recovery of $10-\mathrm{dB}$ or above in the mean level of five frequencies tested in pure-tone audiometry (PTA) at 12 and 24 weeks after the test treatment. The null hypothesis was that $33 \%$ of patients would show hearing improvement, as was reported for a historical control after hyperbaric oxygen therapy (15). At 12 weeks after treatment, $48 \%$ of patients showed hearing improvement, and this proportion increased to $56 \%$ at 24 weeks, which was statistically significant (14). These findings indicate that topical IGF1 application may be efficacious for hearing recovery in patients with SSHL refractory to systemic steroids. However, details of audiometric alterations following topical IGF1 treatment have not yet been elucidated, and might prove crucial for understanding mechanisms of IGF1 therapeutic effects on SSHL and for the design of a consecutive randomized trial. The present study therefore aimed to understand these details. 


\section{MATERIALS AND METHODS}

\section{Patients}

Medical records were reviewed of 25 patients (13 females and 12 males, of median age 49 years at registration [range, 23-72 years]) with SSHL who received topical IGF1 treatment in our previous clinical trial (14). Briefly, the main inclusion criteria of our previous clinical trial were as follows: diagnosis of SSHL within 29 days of onset at the Department of Otolaryngology, Head and Neck Surgery of Kyoto University Hospital, Kyoto, Japan, and hearing recovery of less than 10-dB for an average of PTA thresholds at five frequencies $(0.25,0.5,1,2$ and $4 \mathrm{kHz})$ after systemic steroid treatment. We excluded patients with active chronic otitis media, acute otitis media, otitis media with effusion or dysfunction of the auditory tube, malignant tumors, severe liver dysfunction (aspartate aminotransferase [AST] $>100$ and alanine aminotransferase [ALT] >100), uncontrolled diabetes (haemoglobin A1C [HbA1c] >10), pituitary or adrenal dysfunction, severe systemic illness that affected life expectancy, a history of severe drug allergy, or a history of alcohol or drug dependence within the past 1 year, and pregnant or lactating women. All patients registered in this study had no previous history of noise exposure. This study was conducted in accordance with the Declaration of Helsinki and its amendments, and approved by the Ethical Committee of the Graduate School of Medicine, Kyoto University (registered number, C165). Each patient gave written, informed consent to participate in this study.

\section{Intervention}

Patients received topical IGF1 treatment using gelatin hydrogels at a median of 23 days 
(range 15-32 days) after the onset of SSHL, between 2007 and 2009 at Kyoto University Hospital. Gelatin hydrogels were made from porcine skin gelatin (Nitta Gelatin Inc., Osaka, Japan) in a clean room at the Department of Pharmacy, Kyoto University Hospital. Topical IGF1 treatment procedures were performed in the Day-Surgery Unit of Kyoto University Hospital. Mecasermin (recombinant human IGF1, Somazon ${ }^{\circledR}, 10$ mg for injection; Astellas Pharma Inc., Tokyo, Japan) was dissolved in physiological saline at a final concentration of $10 \mathrm{mg} / \mathrm{ml}$. A 30- $\mu \mathrm{l}$ sample of mecasermin solution was mixed with $3 \mathrm{mg}$ gelatin hydrogels 60 min before application. The single treatment involved placing hydrogel containing $300 \mu \mathrm{g}$ mecasermin in the round-window niche of the middle ear following tympanostomy under local anesthesia with $1 \%$ lidocaine. Patients were hospitalized for four days after the surgical procedure, and their general and local conditions were examined at the outpatient clinic of the Department of Otolaryngology, Head and Neck Surgery, Kyoto University Hospital, for 24 weeks after the test treatment.

\section{Audiometric examination}

PTA was performed on the day of registration and at one, two, four, 12 and 24 weeks after the test treatment. Thresholds in air-conductance at frequencies of $0.25,0.5,1,2$, and $4 \mathrm{kHz}$ were determined.

\section{Outcome measures}

A retrospective chart review was performed with a primary outcome measure of 
alterations in PTA thresholds for each frequency tested and for the average of five frequencies during the observation period. Secondary outcome measures included differences in final improvements of PTA thresholds among frequencies tested, and cumulative numbers of patients showing 10 - or 20 -dB recovery in the average of PTA thresholds at each time-point following treatment.

\section{Statistical analysis}

Alterations in PTA thresholds during the observation period and differences in final improvements of PTA thresholds among frequencies tested were examined by the repeated measurement analysis of variance (ANOVA). The final recovery in PTA thresholds following topical IGF1 treatment was compared with that in a historical control of hyperbaric oxygen therapy for 199 patients with glucocorticoids-resistant SSHL in Kyoto University Hospital between October, 2000 and September, 2006 (15) by unpaired t-test. $P<0.05$ was considered statistically significant. 


\section{Results}

\section{Alterations in PTA thresholds}

Figure 1 shows alterations in mean PTA hearing levels between at registration and 24 weeks after treatment for each patient. The mean PTA hearing level at registration was $\underline{81.2 \mathrm{~dB}(95 \% \text { confidence interval [CI], 71.2-91.1) and that of } 24 \text { weeks after treatment }}$ was $69.3 \mathrm{~dB}$ (95\% CI, 59.8-78.7). The time course of alterations in averages of PTA thresholds is shown in Figure 2A and was statistically significant $(P<0.001)$. Figure 2B-F shows the time courses of alterations in PTA thresholds at frequencies of $0.25,0.5$, 1, 2 and $4 \mathrm{kHz}$, which were all found to be statistically significant $(P<0.001)$. These findings indicate that topical IGF1 treatment via gelatin hydrogels has significant effects on the recovery of PTA thresholds in patients with SSHL refractory to systemic steroids.

\section{Difference in PTA recovery among frequencies}

The mean recovery for the average PTA threshold of five frequencies tested 24 weeks after treatment was $11.9 \mathrm{~dB}$ (95\% CI, 5.8-17.9), and means of recoveries in PTA thresholds for each frequency are shown in Figure 3. Differences in the recovery of PTA thresholds among frequencies were statistically significant $(P=0.0003)$, and we observed a trend that hearing recovery at 2 or $4 \mathrm{kHz}$ was smaller than at lower frequencies. These findings indicate that PTA hearing recovery following topical IGF1 treatment predominantly occurred at low frequencies.

\section{Time-points for hearing recovery}


Cumulative numbers of patients showing 10 - or 20-dB recovery in PTA during the observation period are shown in Fig. 4. For the average PTA threshold of five frequencies tested, 14 patients showed 10-dB recovery and eight showed 20-dB recovery at the end of the observation period. Four weeks after treatment, $10-\mathrm{dB}$ recovery was found in 11 patients (79\%) and 20-dB recovery in four patients (50\%) (Fig. 4). For each frequency, 10 -dB recovery at 4 weeks was found in $17 / 19$ patients (89\%) at $0.25 \mathrm{kHz}, 17 / 19(89 \%)$ at $0.5 \mathrm{kHz}, 13 / 19(68 \%)$ at $1 \mathrm{kHz}, 12 / 14(86 \%)$ at $2 \mathrm{kHz}$ and 8/12 (67\%) at $4 \mathrm{kHz}$ (Fig. 3B-F). At 4 weeks, 20-dB recovery appeared in 7/11 patients (64\%) at $0.25 \mathrm{kHz}, 11 / 13(85 \%)$ at $0.5 \mathrm{kHz}, 7 / 10(70 \%)$ at $1 \mathrm{kHz}, 3 / 6(50 \%)$ at $2 \mathrm{kHz}$ and 5/6 (83\%) at $4 \mathrm{kHz}$. These findings demonstrate that PTA hearing recovery after topical IGF1 treatment occurred during the initial four weeks in the majority of patients, but that further recovery was evident in some patients at a later stage.

\section{Comparison with a historical control}

The recoveries of PTA thresholds for each frequency tested and for the average of five frequencies 24 weeks after topical IGF1 treatment were compared with those in a historical control of hyperbaric oxygen therapy (Fig. 3). The recovery of the average $\underline{\text { PTA thresholds after topical IGF1 treatment was larger than that of a historical controls }}$ $(11.9 \pm 2.9$ [mean \pm standard error] for IGF1 v.s. $8.0 \pm 0.9$ for historical control $)$, but the difference was not statistically significant $(P=0.08)$. For each frequency, there is a trend that topical IGF1 treatment shows better PTA threshold recoveries than a historical control (Fig. 3). The difference in PTA threshold recoveries at $1 \mathrm{kHz}$ between the two group was statistically significant at $P=0.04$, while at other frequencies no 
significant differences in PTA threshold recoveries were observed. 


\section{Discussion}

The present study demonstrates that topical IGF1 treatment has significant effects on the PTA thresholds of patients with SSHL refractory to systemic steroids. Topical IGF1 application using gelatin hydrogels was performed at 23 days after SSHL onset, and led to an average recovery of $11.9 \mathrm{~dB}$ in PTA thresholds over five frequencies. $\underline{\text { In }}$ comparison with a historical control of hyperbaric oxygen therapy, there is a trend that topical IGF1 treatment shows better hearing recovery than hyperbaric oxygen therapy, and a significant difference in hearing recovery is found at $1 \mathrm{kHz}$. After topical IGF1 treatment, recovery at or above $10 \mathrm{~dB}$ was found at $0.25,0.5$ and $1 \mathrm{kHz}$ frequencies. Maximum recovery of $16.0 \mathrm{~dB}$ occurred at $0.5 \mathrm{kHz}$, while hearing recovery at 2 or 4 $\mathrm{kHz}$ was less than $10 \mathrm{~dB}$, suggesting that irreversible degeneration might occur in the high frequency region of cochleae. On the other hand, significant hearing recovery was also observed at low frequencies, suggesting that damage in the low frequency region $\underline{\text { may be reversible and respond to IGF1 delivered by gelatin hydrogels from the round }}$ window.

Regarding the time course of alterations in PTA thresholds for the average of five frequencies, hearing recovery was initiated two weeks after treatment and had stabilized by four weeks, which corresponds to recovery starting approximately five weeks after the onset of SSHL symptoms and continuing until seven weeks after the onset. In general, spontaneous SSHL recovery has been shown to occur between days 3-14 after its onset $(1,2)$, so the PTA recovery observed in the present study may not be spontaneous. PTA thresholds at $1 \mathrm{kHz}$ exhibited a trend of linear recovery during the observation period, while those at other frequencies and alterations in cumulative numbers of patients with a $10 \mathrm{~dB}$ recovery showed a similar trend to the average of all 
five frequencies. An increase in cumulative numbers of patients showing $20 \mathrm{~dB}$ recovery was slightly delayed in comparison with that showing $10 \mathrm{~dB}$ recovery, which initiated at four weeks after treatment and continued to 12 weeks. In short, hearing recovery following topical IGF1 treatment appeared to take approximately four weeks, and, in cases with good recovery, further delayed recovery occurred.

Previous animal experiments have demonstrated crucial roles of IGF1 in the physiolopathology of hearing $(16,17)$. We have also revealed that IGF1 has otoprotective effects against noise- and ischemia-induced cochlear damage using in vivo animal models (11-13). As for mechanisms of hearing recovery by IGF1, two possible explanations are aroused. One possible mechanism is the rescue of cochlear cells from cell death via IGF1 receptor-mediated actions, which has been indicated by animal experiments. IGF1 binding to IGF1 receptors activates several intracellular signaling pathways associated with the promotion of cell survival (18). Another is the regulation of glucose transport in cochlear cells. IGF1 also has insulin-like actions including promoting glucose transport (19), which can be associated with the survival of cochlear hair cells $(20,21)$. Both mechanisms might be involved in hearing recovery by topical application of IGF1. Further studies are required for elucidation of detailed mechanisms.

Recently, the intratympanic injection of steroids has gained considerable attention as an alternative for systemic steroids that may reduce the risk of developing systemic side effects and enables the delivery of more steroids into the cochlea. Rauch et al. reported that the intratympanic injection of steroid effects as an initial treatment for SSHL are not inferior to those of systemic steroids (22), and there is an increasing number of case series evaluating intratympanic steroids as a salvage therapy for SSHL refractory to 
systemic steroids (23-28). Table 1 shows a summary of six studies, of which evidence levels are superior to IV, and of which sample sizes are larger than 20 subjects. The median PTA recovery obtained in these studies is $14.0 \mathrm{~dB}$ (range, 5-22 dB), which is in agreement with that obtained in the present study.

Of previous studies on intratympanic steroids as salvage treatment, two describe detailed time courses of PTA thresholds. Ho et al. (29) reported a time course of alterations in PTA thresholds following the intratympanic injection of dexamethasone. Four weeks after the initiation of treatment (approximately six weeks after the onset), an average recovery of $24.3 \mathrm{~dB}$ was reported, with little further improvement. Similarly, Kilic et al. (26) examined PTA thresholds for an average of four frequencies following intratympanic injections of methylprednisolone every three days for a total of five applications. At four weeks after the initiation of treatment (approximately eight weeks after the onset), an improvement of $19.8 \mathrm{~dB}$ was found, with no further improvements occurring during the observation period. The time course of hearing recovery after topical IGF1 treatment therefore appears to be similar to that which occurs after intratympanic steroid application.

\section{Conclusions}

The present results indicate that topical IGF1 application via gelatin hydrogels may be efficacious for SSHL refractory to systemic steroids. Although the level and time course of hearing recovery following topical IGF1 treatment is similar to that following IT steroids, the baselines of PTA thresholds and methods for evaluation vary among studies. In addition, pharmacological actions of IGF1 differ from those of steroids. Therefore, 
the difference in therapeutic potential for SSHL refractory to systemic steroids between topical IGF1 and intratympanic steroid treatment is unclear. To clarify this, we are currently performing a randomized trial to compare the efficacy of topical IGF1 treatment with that of intratympanic steroids for SSHL refractory to systemic steroids, which may provide critical information on salvage treatments for refractory SSHL. 


\section{References}

1. Mattox DE, Simmons FB. Natural history of sudden sensorineural hearing loss. Ann Otol Rhinol Laryngol 1977; 86: 463-80.

2. Byl FM. Sudden hearing loss: eight years' experience and suggested prognostic table. Laryngoscope 1984; 94:647-61.

3. Schreiber BE, Agrup C, Haskard DO, et al. Sudden sensorineural hearing loss. Lancet 2010;375:1203-11.

4. Rauch SD. Clinical practice. Idiopathic sudden sensorineural hearing loss. N Engl J Med. 2008;359:833-40.

5. Wilson WR, Byl FM, Laird N. The efficacy of steroids in the treatment of idiopathic sudden hearing loss. A double-blind clinical study. Arch Otolaryngol 1980;106: 772-6.

6. Wei BP, Mubiru S, O'Leary S. Steroids for idiopathic sudden sensorineural hearing loss. Cochrane Database Syst Rev 2006;1:CD003998.

7. Murillo-Cuesta S, Rodríguez-de la Rosa L, Cediel R, et al. The role of insulin-like growth factor-I in the physiopathology of hearing. Front Mol Neurosci. 2011;4:11.

8. Salt AN, Plonkte SK. Principles of local drug delivery to the inner ear. Audiol Neurotol 2009;14:350-60.

9. Young S, Wong M, Tabata Y, et al. Gelatin as a delivery vehicle for the controlled release of bioactive molecules. J Control Release 2005;109: 256-74.

10. Endo T, Nakagawa T, Kita T, et al. A novel strategy for treatment of inner ears using a biodegradable gel. Laryngoscope 2005;115: 2016-20.

11. Iwai K, Nakagawa T, Endo T, et al. Cochlear protection by local IGF-1 application using biodegradable hydrogel. Laryngoscope 2006;116: 526-33. 
12. Lee KY, Nakagawa T, Okano T, et al. Novel therapy for hearing loss: Delivery of insulin-like growth factor-1 to the cochlea using gelatin hydrogel. Otol Neurotol 2007;28: 976-81.

13. Fujiwara T, Hato N, Nakagawa T, et al. IGF1 treatment via hydrogels rescues cochlear hair cells from ischemic injury. Neuroreport 2008;19: 1585-8.

14. Nakagawa T, Sakamoto T, Hiraumi H, et al. Topical insulin-like growth factor-1 treatment using gelatin hydrogels for glucocorticoid-resistant sudden sensorineural hearing loss: a prospective clinical trial. BMC Med 2010;8:76.

15. Miura M, Sakamoto T, Hiraumi H, et al. Evaluation of hyperbaric oxygen therapy for the treatment of sudden hearing loss in both primary and secondary cases. Practica Oto-Rhino-Laryngologica 2008; 101: 749-57.

16. Murillo-Cuesta S, Rodríguez-de la Rosa L, et al. The role of insulin-like growth factor-I in the physiopathology of hearing. Front Mol Neurosci. 2011;4:11.

17. Okano T, Xuan S, Kelley MW. Insulin-like growth factor signaling regulates the timing of sensory cell differentiation in the mouse cochlea. J Neurosci. 2011; 31:18104-18.

18. Aburto MR, Magariños M, Leon Y, et al. AKT signaling mediates IGF-I survival actions on otic neural progenitors. PLoS One 2012;7:e30790.

19. Metzger F, Sajid W, Saenger S, et al. Separation of fast from slow anabolism by site-specific PEGylation of insulin-like growth factor I (IGF-I). J Biol Chem. 2011; 286:19501-10.

20. Lim DJ. Effects of noise and ototoxic drugs at the cellular level in the cochlea: a review. Am J Otolaryngol 1986;7:73-99.

21. Olmos PR, Borzone GR, Olmos JP, et al. Mitochondrial diabetes and deafness: 
possible dysfunction of strial marginal cells of the inner ear. J Otolaryngol Head Neck Surg 2011; 40:93-103.

22. Rauch SD, Halpin CF, Antonelli PJ, et al. Oral vs intratympanic corticosteroid therapy for idiopathic sudden sensorineural hearing loss: a randomized trial. JAMA. 2011;305:2071-9

23. Plontke S, Lowenheim H, Preyer S, et al. Outcomes research analysis of continuous intratympanic glucocorticoid delivery in patients with acute severe to profound hearing loss: basis for planning randomized controlled trials. Acta Otolaryngol 2005; 125: 830-9.

24. Choung YH, Park K, Shin YR, et al. Intratympanic dexamethasone injection for refractory sudden sensorineural hearing loss. Laryngoscope 2006; 116: 747-52.

25. Roebuck J, Chang CYJ. Efficacy of steroid injection on idiopathic sudden sensorineural hearing loss. Otolaryngol Head Neck Surg 2006; 135: 276-9.

26. Kilic R, Safak MA, Oquz H, et al. Intratympanic methylprednisolone for sudden sensorineural hearing loss. Otol Neurtol 2007; 28: 312-6.

27. Haynes DS, O’Malley M, Cohen S, et al. Intratympanic dexamethasone for sudden sensorineural hearing loss after failure of systemic therapy. Laryngoscope 2007;117: 3-15.

28. Kakehata S, Saski A, Futai K, et al. Daily short-term intratympanic dexamethasone treatment alone as an initial or salvage treatment for idiopathic sudden sensorineural hearing loss. Audiol Neurotol 2011;16:191-7.

29. Ho HG, Lin HC, Shu MT, et al. Effectiveness of intratympanic dexamethasone injection in sudden-deafness patients as salvage treatment. Laryngoscope 2004;114: 1184-9. 


\section{Figure Legends}

Fig. 1. Alterations in hearing levels in pure tone audiometry (PTA) between at registration (regist.) and 24 weeks after topical IGF1 treatment in each patients.

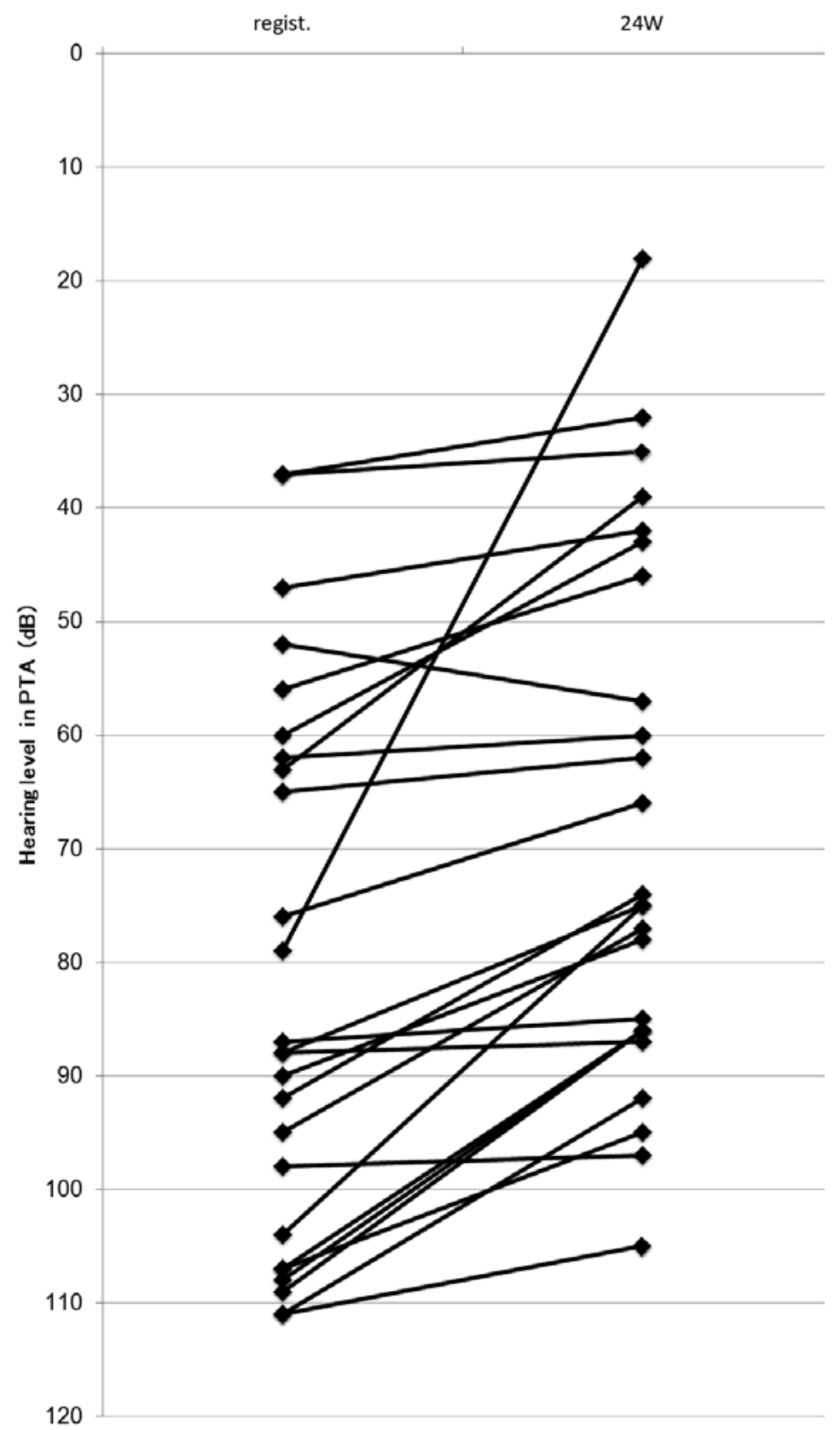

Fig. 2. Hearing levels in pure tone audiometry (PTA) at registration (regist.) and following topical IGF1 treatment, in the average of five frequencies tested (A), at 0.25 
kHz (B), $0.5 \mathrm{kHz}$ (C), $1 \mathrm{kHz}$ (D), $2 \mathrm{kHz}$ (E) and 4 kHz (F). Bars represent standard errors.
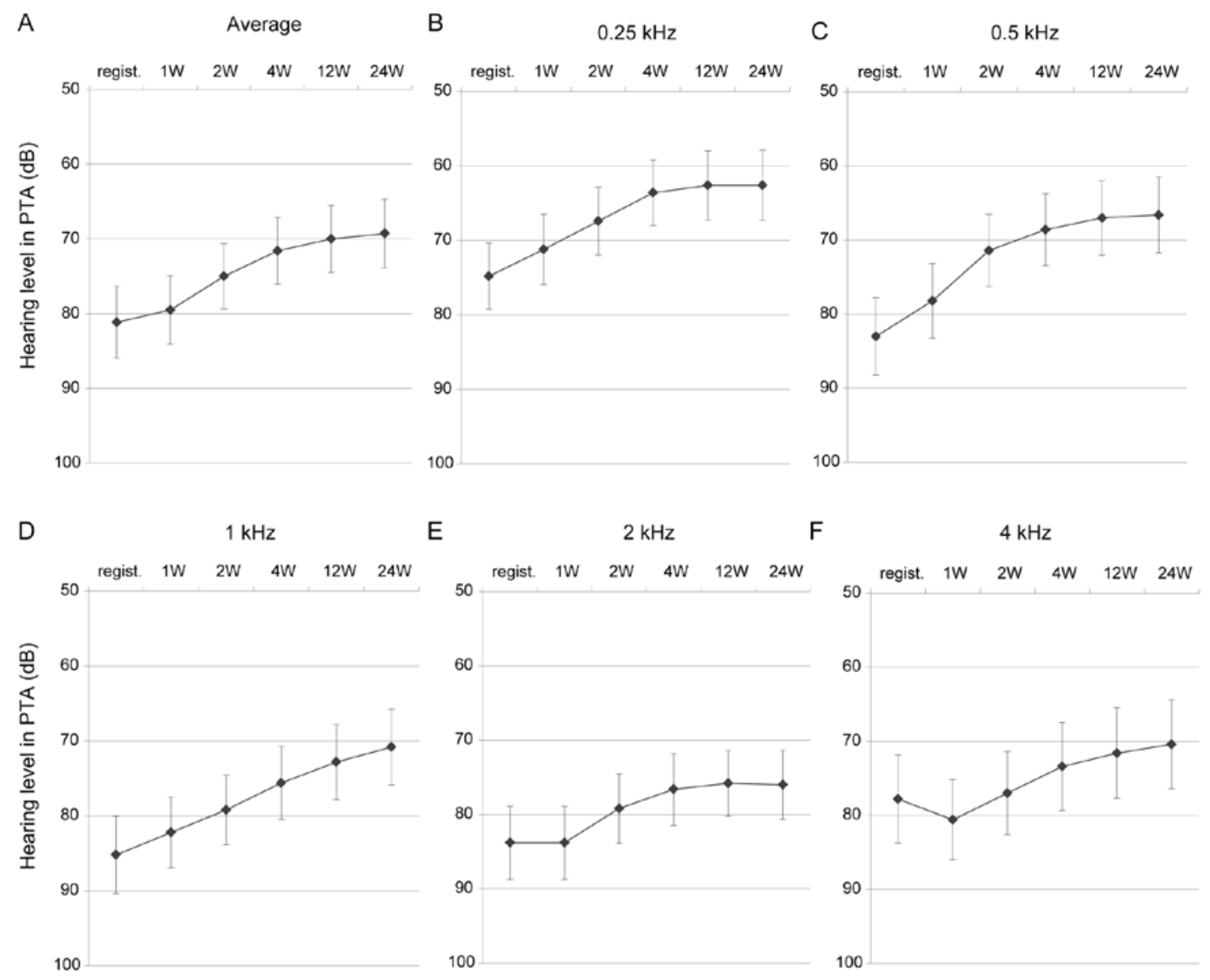

Fig. 3. Final hearing recovery in the average of five frequencies tested and at each frequency tested after topical IGF1 treatment or in a historical control of hyperbaric oxygen therapy (HBO). An asterisk indicates a significant difference in hearing recovery between topical IGF1 treatment and HBO. Bars represent standard errors. 


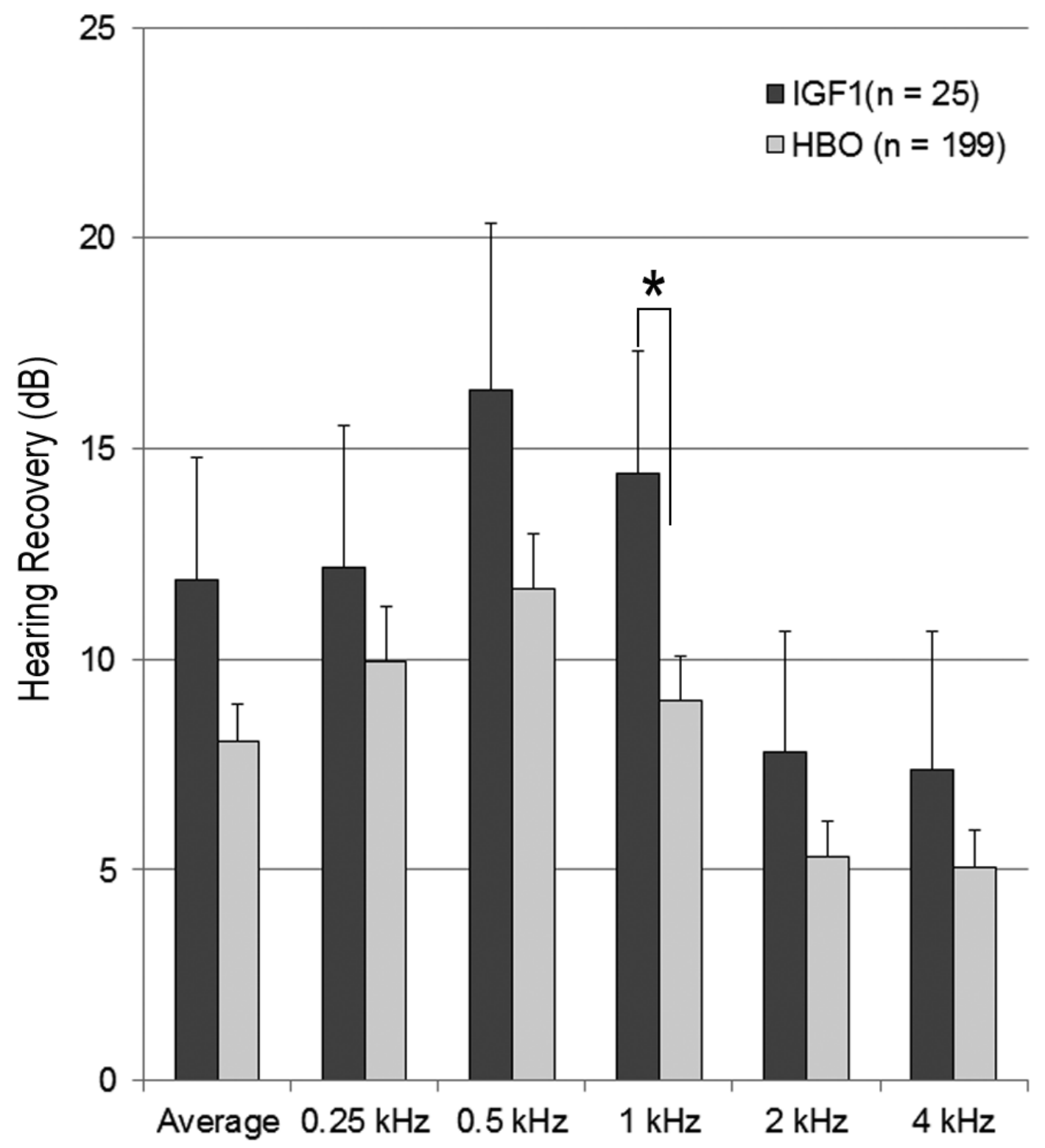

Fig. 4. Cumulative numbers of patients showing $10-$ or $20-\mathrm{dB}$ recovery in the average of five frequencies during the observation period. Bars represent standard errors. 


\section{Average}

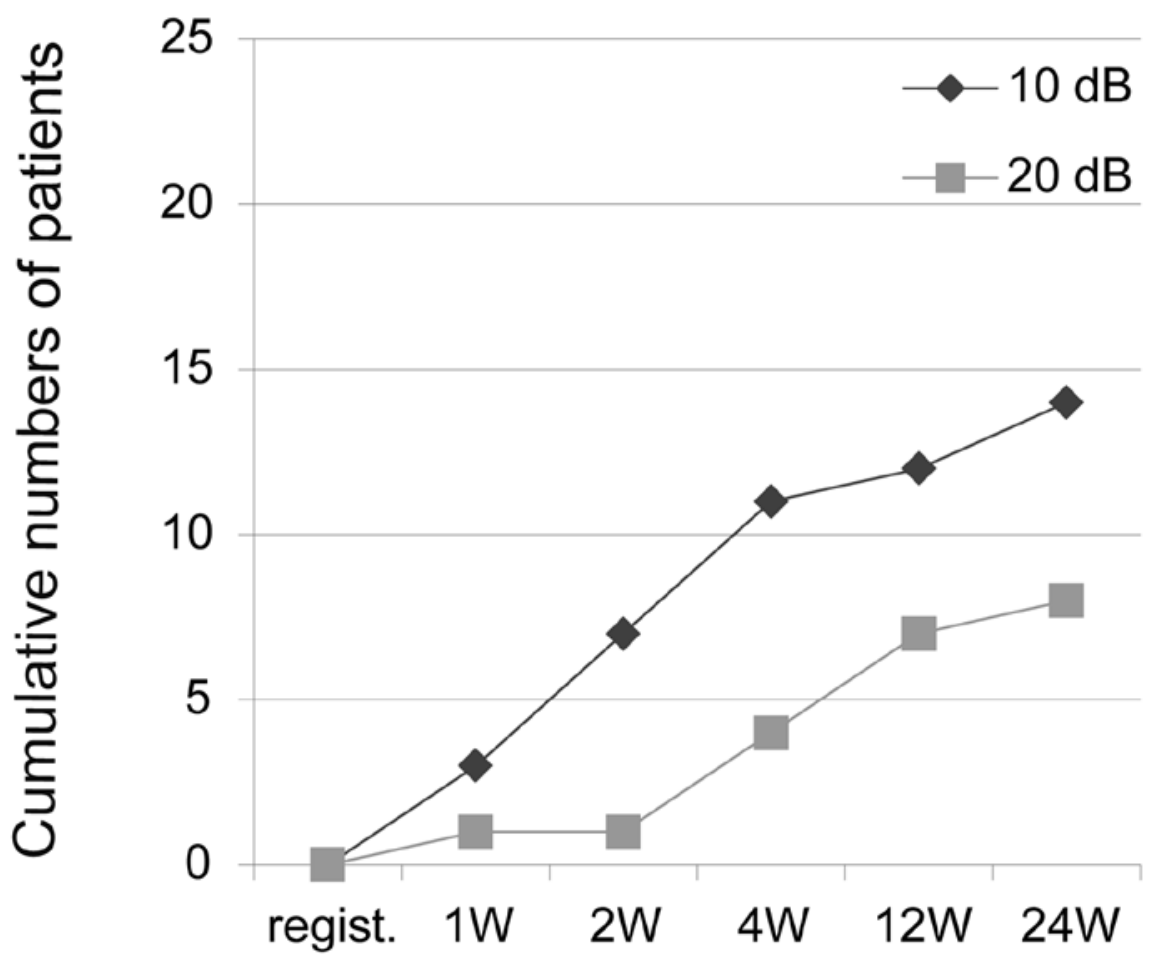

Table 1. Audiometric outcomes in studies of the intratympanic injection of steroids for sudden sensorineural hearing loss refractory to systemic steroids. PTA: pure tone audiometry, RW: round window, Dex: dexamethasone, Mp: methylprednisolone, NA: not available.

\begin{tabular}{|c|c|c|c|c|c|}
\hline First author & Sample size & $\begin{array}{l}\text { Steroid, } \\
(\mathrm{mg} / \mathrm{ml})\end{array}$ & Treatment protocol & PTA baseline (dB) & $\begin{array}{l}\text { Hearing improvement } \\
\text { (dB) }\end{array}$ \\
\hline Plontke, 2005 [17] & 23 & Dex (4) or Mp (40) & $\begin{array}{l}\text { RW microcatheter for } 4 \\
\text { weeks }\end{array}$ & 103 & 16 \\
\hline Choung, 2006 [18] & 34 & Dex (5) & $\begin{array}{l}2 \text { injections/week for } 2 \\
\text { weeks }\end{array}$ & 72 & 9 \\
\hline Roebuck, 2006 [19] & 31 & Dex (24) & 1 injection & 69 & 12 \\
\hline Kilic, 2007 [20] & 20 & Мp (62.5 & $\begin{array}{l}5 \text { injections at 3-day } \\
\text { intervals }\end{array}$ & 65 & 22 \\
\hline Haynes, 2007 [21] & 40 & Dex (24) & 1 injection & NA & 5 \\
\hline Kakehata, 2011 [22] & 24 & Dex (4) & 1 injection/day for 8 days & 75 & 17 \\
\hline
\end{tabular}

